L'incertitude dans l'époque du passage au périhélie ne monte pas à \pm 3 jours.

La comète restera extrêmement faible pendant toute cette apparition. Au maximum de son éclat elle aura la même intensité lumineuse que vers le 2 décembre 1895 , époque à laquelle elle ne fût plus observée qu'aux observatoires de Northfield, Charlottesville, Denver et Mount Hamilton. On peut néanmoins espérer qu'on retrouvera la comète et qu'on l'observera aux instruments les plus puissants, vu

Paris, le 2 I mai I 902 . que l'incertitude de sa position n'est pas considérable et qu'en 1896 MM. Campbell et Hussey ont pu encore l'observer le 5 février lorsque $1: r^{2} \Delta^{2}$ n'était que de 0.048 .

Dans les trois prochaines apparitions la comète sera encore beaucoup plus faible qu'en rgo2. Il est donc fort désirable de l'observer dans cette apparition pour pouvoir assurer sa découverte dans l'apparition de r93 1 qui sera aussi favorable que celle de 1895 .

\title{
Beobachtungen des Cometen 1902 a
}

am 6 zöll. Steinheil'schen Refractor der Sternwarte Pola der k. u. k. Kriegs-Marine.

\begin{tabular}{|c|c|c|c|c|c|c|c|c|c|c|c|}
\hline $\begin{array}{l}1902 \\
\text { pril } 17\end{array}$ & $\begin{array}{l}\text { M. Z. Pola } \\
\text { I }^{\mathrm{h}} 27^{\mathrm{m}} 2^{\mathrm{s}}\end{array}$ & $\begin{array}{c}\Delta \alpha \\
-1^{m_{26.92}}\end{array}$ & $\begin{array}{c}\Delta \delta \\
+2^{\prime} 2 " 0\end{array}$ & $\begin{array}{l}\text { Vgl. } \\
3\end{array}$ & Mikr. & $\begin{array}{c}\alpha \text { app. } \\
23^{\mathrm{h}} \stackrel{28^{\mathrm{m}}}{3} 39^{\mathrm{s}} \cdot 5^{8}\end{array}$ & $\begin{array}{c}\log p . \Delta \\
9.657 \mathrm{n}\end{array}$ & $\begin{array}{r}8 \text { app. } \\
+23^{\circ} 55\end{array}$ & $6 " 6$ & $\begin{array}{r}\log p .4 \\
0.769\end{array}$ & $\begin{array}{l}\text { Red. ad l. app. } \\
+0.52-0 . " g\end{array}$ \\
\hline I & 324 & & & & $\mathbf{R}$ & & & & & & \\
\hline
\end{tabular}

Beobachter: Linienschiffsfähnrich Rudolf Höhl.

April 17. Comet mit einem Kern rom. Schweif $5^{\prime}$ Länge. - April 18 . Im Anfang der Beobachtung stört untergehender Mond, am Schluss Morgengrauen. Comet scheinbar lichtschwächer, Kern etwa $10^{\mathrm{m}} \cdot 5$ bis $1 \mathrm{I}^{\mathrm{m}}$.

$$
\begin{aligned}
& \text { Mittlere Oerter der Vergleichsterne. } \\
& \text { * } \alpha 1902.0 \quad \delta 1902.0 \quad \text { Autorität } \\
& \text { I } 23^{\mathrm{h}} 30^{\mathrm{m}} 5^{\mathrm{s}} .98+23^{\circ} 53^{\prime} 5^{\prime \prime} 5 \% \text { (W. } 593-4+2 \text { AG. Berl. B. 903 I) } \\
& 2234211.50+214347.01 / 2\left(M_{13276 I}+\right.\text { AG. Berl. B. 9090) }
\end{aligned}
$$

Pola, 1902 April 22.

Der Vorstand: $K$. Koss, k. u. k. Corvettencapitän.

\section{Literarische Anzeigen.}

A. Krisch. Astronomisches Lexicon. Vollständig in 20 Lieferungen. Wien, A. Hartleben's Verlag. 1902.

Soweit die bisher erschienenen ro Lieferungen es beurtheilen lassen, wird das Lexicon seinen Zweck, ein bequemes Nachschlagebuch für den sich für Astronomie Interessirenden zu bilden, erfüllen. Die Reichhaltigkeit des Inhalts und vor allem die schönen Abbildungen sind anzuerkennen; im Einzelnen wird allerdings der Fachastronom manche Ausstellungen zu machen und besonders an den zahlreichen Daten und Tabellen vieles zu verbessern haben.

Ludolf Camphausen's Leben. Nach seinem schriftlichen Nachlass dargestellt von Anna Caspary. Stuttgart und Berlin, J. G. Cotta'sche Buchhandlung. 1902.

Die Thätigkeit Camphausen's als Astronom nach seinem Rücktritte aus dem Staatsdienst, vgl. den Nachruf von H. C. Vogel in Nr. 3021 , wird einen Hinweis auf das vorliegende Buch an dieser Stelle rechtfertigen. Besonders in den beiden letzten Capiteln befinden sich viele interessante Notizen über Camphausen's wissenschaftliche Arbeiten und seinen Verkehr mit zeitgenössischen Gelehrten.

W. F. Wislicenus. Astronomischer Jahresbericht. Herausgegeben mit Unterstuitzung der Astronomischen Gesellschaft. III. Band, enthaltend die Literatur des Jahres 1901. Berlin, Verlag von Georg Reimer. I902.

Die Raschheit des Erscheinens, die geschickte Redaction der Referate und die Vollständigkeit des Gebotenen können auch diesmal nur rühmend hervorgehoben werden. Nur iber einen Punkt, wie weit sich nämlich die Referate zu erstrecken haben, befinde ich mich nicht ganz im Einverständniss mit dem Herrn Verfasser. Nach meiner Ansicht verdienen die zahlreichen astronomischen Notizen, die sich in den populären naturwissenschaftlichen Zeitschriften eingestreut finden, nur selten die Aufnahme in den Jahresbericht. Wenn in diesem Punkt der Verfasser seinen allzu objec. tiven Standpunkt verlassen und nur eine Auswahl treffen wollte, so würde dies sicher dem Bericht zum Vortheil gereichen.

F. K. Ginze1. Die astronomischen Kenntnisse der Babylonier und ihre culturhistorische Bedeutung. 3 Aufsätze. Sonderabdruck aus C. F. Lehmann's Beiträgen zur alten Geschichte I. Leipzig 1902.

Die Abhandlung giebt einen Ueberblick uber den heutigen Stand der Kenntniss der babylonischen Astronomie und wird auch von den Astronomen mit grossem Interesse gelesen werden.

Inhalt zu Nr. 3794. L. Courvoisier. Ueber ein neues Beobachtungsverfahren mit dem Registrirmikrometer. I7. - H. Struve. Ueber die Doppelsternmessungen am grossen Pulkowaer Refractor. 19. - E. C. Pickering. A new Algol variable 10.1902 Cygni. 23. E. Millosevich. Sull' effemeride rigorosa di (433) Eros. 25. - A. R. Hinks. Note on one of the stars selected as an 'étoile de repère for the reduction of photographs of Eros. 27. - W. Valentiner. Beobachtungen des Planeten I9o2 HP. 27. M. Wolf. Photographische Beobachtungen von kleinen Planeten. 27. - L. Schulhof. Eléments et éphéméride de recherche de la comète 1895 II (Swift). 29. - K. Koss. Beobachtungen des Cometen I902 a. 31. - Literarische Anzeigen. 3 I. 